\title{
Thoracic empyema: a study of 56 patients
}

\author{
E D O Mangete, B B Kombo, T E Legg-Jack
}

\begin{abstract}
Fifty seven children with thoracic empyema ( 37 boys and 20 girls) aged less than 12 years were seen at the University of Port Harcourt Teaching Hospital between January 1989 and December 1991. Staphylococcus aureus was the most common organism isolated from the pus of these patients (36 $(63 \%)$ patients). Pseudomonas aeruginosa, the next most common organism, was isolated in $10(18 \%)$ patients. The most common symptoms at presentation were acute illness with fever and cough $(51(89 \%)$ patients). All the patients were treated with closed intercostal tube drainage and appropriate antibiotics. Decortication was resorted to in only one patient. There were two deaths and the overall survival rate was $\mathbf{9 7 \%}$. (Arch Dis Child 1993; 69: 587-588)
\end{abstract}

Thoracic empyema, the collection of pus in the pleural space, though decreasing in many cases, still contributes significantly to mortality and morbidity of children in the less developed countries. $^{12}$ We report here 56 children with thoracic empyema seen at the University of Port Harcourt Teaching Hospital over a three year period from January 1989 to December 1991. A further subject, a 10 year old boy, was discharged against medical advice before treatment was completed and could not be included in the study.

\section{Subjects and methods}

This was a prospective study and the protocols were completed for each child with empyema aged less than 12 years. At the end of the study period all the completed protocol sheets and the casenotes of these children were reviewed with regard to aetiology, clinical symptoms, bacteriology, social class, mode of treatment, and eventual outcome of treatment.

The diagnoses were initially based on clinical findings or chest radiographs and were confirmed by needle aspiration of the pus and bacteriological examination of the aspirate. Treatment with broad spectrum antibiotics began once sufficient aspirate was obtained for microscopy, culture, and sensitivity tests. Antibiotic treatment was altered, if necessary, when the results of the sensitivity tests were available. Closed thoractomy tube drainage was carried out in all patients once the pus had been aspirated during thoracocentesis.

\section{University of Port}

Harcourt Teaching

Hospital, PMB 6173, Port

Harcourt, Nigeria

E D O Mangete

B B Kombo

T E Legg-Jack

Correspondence to:

Dr Mangete.

Accepted 11 March 1993
Results

Forty five (80\%) children belonged to social class III (labourers, manual workers, unemployed, living in shanty settlements), whereas $11(20 \%)$ belonged to social class II (clerks, lower cadre

officers in government or private companies living in one or two bedroomed flats in high density areas). None of the patients belonged to social class I (managers and higher cadre officers in government or private companies living in low density areas or self contained flats in moderately populated areas). The youngest child was 9 weeks old and the oldest 11 years 10 months. The peak incidence for boys and girls was in the 0-3 year age group.

The most common clinical presentation was acute illness with fever and cough (51 (91\%) patients). Dypsnoea with intercostal muscle retraction and tachypnoea (respiratory rate $\geqslant 50$ / minute) was found in 41 (73\%) patients. Only three $(5 \%)$ children presented in the chronic phase with low grade fever and weight loss. All three children presenting in the chronic phase were older than 10 years. The right side was affected more often ( 33 children $(59 \%)$ ) than the left side ( 23 children $(41 \%)$ ).

A moderate to marked degree of anaemia, leucocytosis, and increased erythrocyte sedimentation rate were observed in all patients.

Blood films were examined for malaria parasites in $31(55 \%)$ children; malaria parasites were present in only three. Medical histories indicated a high incidence of frequent malaria attacks in these children, however. Slight to moderate eosinophilia was observed in eight (14\%) children and ascaris worms were found in the stools of five (9\%) patients. Pneumothorax with bronchial fistula was present in two children and toxic myocarditis was diagnosed in five.

Apart from two of the patients who presented in the chronic phase, medical advice was sought within the first week of onset of acute illness.

Microscopy, culture, and sensitivity studies of the aspirated pus was carried out in all 56 patients. The most common bacterium isolated from the pleural aspirate was Staphylococcus aureus.

Blood culture was carried out only on the two patients who presented with symptoms of generalised septicaemia; the blood cultures were sterile. The chest radiographs showed opacity consistent with pyothorax in 54 (96\%). Six children had associated cardiomegaly. Nonspecific pulmonary infection was the most common cause and was present in $49(88 \%)$ of the patients.

Empyema resulting from pulmonary tuberculosis was found in only two patients. No empyema of traumatic origin was seen in this study.

TREATMENT AND RESULTS OF TREATMENT

All 56 patients were treated intravehously with systemic antibiotics after sufficient pus had been aspirated for the culture and sensitivity tests. Alterations in the antibiotic treatment were 
carried out based on the results of the sensitivity tests. Most of the patients were treated with a combination of cloxacillin, ampicillin, and gentamicin. Cefotaxime was the drug of choice in the treatment of four of the patients. The two patients with pulmonary tuberculosis were treated with thiacetazone/isoniazid, rifampicin, and streptomycin. These patients were closely monitored for side effects of these drugs. All the patients had closed thoracostomy tube drainage. Argyle chest tubes (sizes Charriere 16 and 18) were used, the size depending on the age of the patient. The tubes were inserted under local anaesthesia at the sixth intercostal space in the mid axillary line. In $35(63 \%)$ patients the thoracotomy drainage tube was removed within the first 14 days of insertion. Only five patients required drainage beyond five weeks. Two patients died during treatment; one died of septicaemia and the other presented on admission in a poor nutritional state and died two days after admission. Mixed bacilli ( $S$ aureus and $P$ seudomonas aeruginosa) were cultured from the pus of this patient. One patient had decortication for thickened pleura, which prevented lung expansion. The postoperative period was uneventful and lung expansion thereafter was satisfactory.

The histology of the excised pleura showed non-specific inflammatory changes. Fifty four $(96 \%)$ patients recovered satisfactorily.

\section{Discussion}

Thoracic empyema continues to contribute significantly to the mortality rates among the poor in developing countries. Among the affluent in these countries and in the developed world, thoracic empyema continues to decrease steadily and rapidly.

In this study the maximum incidence of this disease was in the age group $0-3$ years $(34(61 \%)$ patients). This agrees with the findings of other workers $^{34}$ and probably coincides with the arrival or pending arrival of a new baby in the family.

Clinical presentation was that of acute illness, pyrexia, cough, and tachynoea. ${ }^{3-6} S$ aureus was the most common organism isolated from the pus obtained at thoracocentesis of these patients. ${ }^{34}$ The next most common organism was $P$ aeruginosa. $S$ aureus and $P$ aeruginosa are usually resistant to most of the common and therefore often abused antibiotics in developing countries. Empyema resulting from acid fast bacilli were observed in two (3\%) patients, though no case of thoracic empyema of traumatic origin was recorded.

Intercostal closed tube drainage and appropriate antibiotics was the regimen most commonly used in the management of these patients. Surgery (decortication) was resorted to only when this regimen did not produce the desired results. ${ }^{257-9}$

The moderate to marked degree of anaemia observed in these patients is most likely to be due to the frequent attacks of malaria. The anaemia could be further aggravated by infestation with helminths such as Ascaris lumbricoides, as was found in five of the patients. Apart from the three patients who presented with chronic disease, and one other patient who was almost marasmic, the other 52 children were clinically well nourished.

Streptococal enzymatic debridement ${ }^{10}$ or thoracocentesis alone without closed tube drainage was not used in this study. The overall success rate was $96 \%$, which compares favourably with the findings of other workers. ${ }^{12}$

The regimen used (closed thoracotomy tube drainage plus appropriate antibiotics), which is simple though not always cheap, is well suited for the management of these patients. The regimen can be safely carried out in almost all secondary health centres. The more complicated cases with bronchial fistula or thickened pleura, which are relatively rare, should be referred to specialised centres for expert management.

1 Middlekamp JN, Pukerson ML, Burford TH. The changing pattern of empyema thoracis in paediatrics. $\mathcal{f}$ Thorac

2 Beg MH, Ahmad SH, Reyazuddin T, Chandra J. The changing pattern of empyema thoracis in paediatrics. f Thorac Cardiovasc Surg 1964; 47: 165-72.

3 Groff DB, Randolf JG, Blades B. Empyema in childhood. FAMA 1966; 195: 572-5.

4 Stiles QR, Lindesmith GG, Tucker BL, et al. Pleura empyema in children. Ann Thorac Surg 1970; 10: 37-40.

5 Cattaneo SM, Kilman JW. Surgical therapy of empyema in children. Arch Surg 1973; 106: 564-7.

6 Samson PC. Empyema thoracis. Essential of present day management. Ann Thorac Surg 1975; 11: 210-12.

7 Samson PC, Burford TH. Total pulmonary decortication: its evaluation and present concept of indications and operative evaluation and present concept of indicatio

technique. F Thorac Surg 1947; 16: 127-30.
8 Cohn LH, Blaisdell EW. Surgical treatment of nontuberculous empyema. Arch Surg 1970; 100: 376-9.

9 Mayo P, McElverin RB. Early thoracotomy for pyogenic empyema. Ann Thorac Surg 1966; 2: 649-52.

10 Tillet WS, Sherry S, Chritenson LR, et al. Streptococcal enzymatic debridement. Ann Surg 1950; 131: 12-6. 